\title{
Culture, immigration et santé
}

La consommation de médicaments chez les Vietnamiens de Montréal

Marie-Eve Blanc et Laurence Monnais

\section{CpenEdition}

Journals

Édition électronique

URL : https://journals.openedition.org/remi/4238

DOI : $10.4000 /$ remi.4238

ISSN : $1777-5418$

Éditeur

Université de Poitiers

Édition imprimée

Date de publication : 1 décembre 2007

Pagination : 151-176

ISBN : 978-2-911627-47-4

ISSN : 0765-0752

Référence électronique

Marie-Eve Blanc et Laurence Monnais, «Culture, immigration et santé », Revue européenne des

migrations internationales [En ligne], vol. 23 - n³ | 2007, mis en ligne le 01 décembre 2010, consulté le

14 avril 2022. URL : http://journals.openedition.org/remi/4238 ; DOI : https://doi.org/10.4000/remi. 4238

Ce document a été généré automatiquement le 14 avril 2022.

(c) Université de Poitiers 


\title{
Culture, immigration et santé
}

\author{
La consommation de médicaments chez les Vietnamiens de Montréal
}

\author{
Marie-Eve Blanc et Laurence Monnais
}

1 Cet article se propose d'enrichir la problématique santé-immigration en dressant un portrait qualitatif nuancé, contextualisé et centré sur une communauté ethnoculturelle (la communauté vietnamienne du Canada et plus spécifiquement de Montréal) des pratiques de pluralisme médicamenteux et des logiques qui les sous-tendent. À travers une analyse de contenu d'entrevues semi-dirigées effectuées en 2002, il s'est agi de mettre au jour la variabilité des pratiques de pluralisme thérapeutique et les logiques les sous-tendant. L'objectif principal d'une telle étude est d'offrir des clés de compréhension fines de l'impact de l'expérience d'immigration sur l'évolution des pratiques de santé d'immigrants reconnus pour disposer d'une culture très éloignée des repères occidentaux et en particulier une histoire et une culture médicales fortes.

2 Abordant le médicament comme un objet social et culturel, nous estimons que sa consommation constitue un phénomène complexe dont l'étude doit prendre en considération un éventail dynamique de facteurs, qu'ils soient d'ordre politique, économique, éthique, social ou culturel. La consommation de médicaments constitue selon nous un révélateur privilégié de la dimension socioculturelle de la santé. Elle met en évidence l'imbrication entre des représentations, individuelles et collectives, de la santé et de la maladie et les comportements de recherche d'aide qui y sont associés (Whyte et coll., 2002 ; Collin et coll., 2005) et ce d'autant plus dans un contexte métissé tel que celui de plusieurs pays à forte immigration, dont le Canada.

\section{Problématique}

\section{Santé et immigration}

3 Depuis une dizaine d'années, la recherche sur la santé des immigrants canadiens s'articule principalement autour des trois constats majeurs. Tout d'abord, à leur arrivée au pays, les immigrants bénéficieraient d'une meilleure santé que les individus nés au Canada. Ceci serait le résultat direct de la politique d'immigration canadienne qui 
repose sur la sélection des immigrants dans une perspective d'employabilité et passe pour ce faire par l'application du critère de "bonne santé ». En conséquence, les individus portés à s'installer au Canada sont généralement en bonne santé et dans la force de l'âge (McDonald et Kennedy, 2004). Pour autant, leur santé se détériorerait au fil des années d'immigration. Cette détérioration se manifesterait par une augmentation de la prévalence de problèmes chroniques, handicaps et habitudes de vie à risque (Chen et coll., 1996). Parmi les facteurs potentiels de détérioration on retrouve en priorité cités : le type d'immigration, particulièrement le statut de réfugié (Bibeau et coll., 1992 ; Beiser et Hyman, 1997) ; le temps d'immigration, jouant sur l'adaptation à l'environnement physique et social du pays d'accueil (Chen et coll., 1996) et donc l'acculturation (Hyman et Dussault, 1991) ; l'ethnicité, lorsqu'elle repose sur des valeurs non occidentales et s'avère très structurée sur le plan social (Bibeau et coll., 1992; Massé, 1995). À ces facteurs s'ajouteraient ceux intervenant dans la détérioration de la santé de l'ensemble de la population: milieu socio-économique défavorisé, augmentation en âge, faible support social, en particulier chez les femmes (Massé, 1995 ; Dunn et Dyck, 2000).

4 Quelques études qualitatives ont par ailleurs insisté sur l'impact d'une sous-utilisation des services hospitaliers, préventifs et en santé mentale par les immigrants (Globerman, 1998; Hyman, 200; Chevalier et Gravel, 2002), ce en corrélation avec d'autres facteurs dont, là encore, le niveau socio-économique (Kronenberg et coll., 2006) et l'augmentation en âge, surtout chez les communautés asiatiques (Lam, 1994 ; Wen et coll., 1996; Guberman et coll., 1997). Si on identifie en parallèle et de façon relativement proéminente des problèmes d'accessibilité aux services conventionnels de santé canadiens associés à des barrières dites culturelles ${ }^{1}$, les études de Jenkins et coll. (1996) et Wen et coll. (1996) suggèrent pourtant que cette sous utilisation des services pourrait être le résultat d'un choix rationnel associé, entre autres, à des représentations socioculturelles (du corps, de la maladie, du professionnel de santé) spécifiques, un choix qui entraînerait d'ailleurs éventuellement un recours à des services de santé parallèles (Hyman, 2001).

5 Reste que les études spécifiquement centrées sur les pratiques de santé des immigrants canadiens s'avèrent peu nombreuses, surtout si l'on s'arrête à la production d'études qualitatives traitant de communautés ethnoculturelles ciblées. Le paradoxe est grand lorsque l'on considère que le Canada enregistre une immigration très forte, de plus en plus éclatée du point de vue des origines et de plus en plus non-occidentale. Ainsi, lors $\mathrm{du}$ dernier recensement national, on a dénombré plus de 200 origines ethniques différentes. Selon Statistique Canada toujours, les Asiatiques représentaient $58 \%$ des immigrants arrivés au Canada entre 1991 et 2001. Les originaires de la Chine continentale (RPC), de l'Inde, des Philippines, d'Hong Kong, du Sri Lanka et de Taïwan représentent plus de $40 \%$ des immigrants arrivés dans la même période (Statistique Canada, 2003).

6 Par ailleurs, rappelons que le Canada promeut une politique de multiculturalisme (Loi sur le multiculturalisme canadien, L.R., 1985, ch. 24 (4e suppl.)) qui incite au respect des cultures minoritaires, dont en matière de santé (Gravel et Battaglini, 2000). À cet égard, la loi fédérale sur les Produits de Santé Naturels (PSN), entrée en vigueur en janvier $2004^{2}$, ne manque pas de souligner l'importance prioritaire de certaines «traditions médicales ", offrant même un cadre réglementaire - et donc un cadre d'accès - à des « remèdes traditionnels » en concordance avec le poids démographique, probablement 
politique et social aussi de plusieurs communautés phares : la communauté autochtone, chinoise et indienne. En outre, la réflexion actuelle des institutions de santé fédérales autour de la structuration d'une offre sanitaire de type intégrative - c'est-à-dire qui permette un choix égalitaire et éclairé entre médecine allopathique et Médecines Alternatives et Complémentaires (MAC) selon la définition donnée par l'Organisation Mondiale pour la Santé (OMS, 2002) - répondant ainsi davantage aux attentes en la matière et, dans une certaine mesure, aux pratiques de la population canadienne nous incite assurément à nous pencher sur les pratiques de santé des immigrants canadiens (Collin et coll., 2005 ; Singh et Levine, 2006).

\section{La consommation de médicaments chez les immigrants}

7 En parallèle, on estime que de 1992 à 2002 les dépenses des ménages canadiens en matière de médicaments ont augmenté de $70 \%$ (Statistique Canada, 2005) plaçant le pays en quatrième position mondiale dans les dépenses en médicaments par habitant (Institut Canadien d'Information en Santé, 2003). Au-delà d'une dénonciation récurrente du coût financier et humain de cette consommation en forte croissance et des pratiques à risques auxquelles elle est associée - surconsommation, interactions médicamenteuses, automédication, inobservance ou encore sur prescription de la part de certains médecins -, les modalités de ce recours au médicament restent à traduire dans leur complexité (Collin et coll., 2005; Collin et coll., 2006). Ce d'autant plus lorsqu'on consulte le plus récent sondage soumis à Santé Canada en mars 2005 (Ipsos Reid, 2005) sur le recours des Canadiens aux PSN. En effet, ce sondage illustre un engouement populaire profond pour les MAC et en particulier les PSN : $71 \%$ de la population du pays y aurait recours, de façon régulière ou occasionnelle. Il explicite aussi un lien fait par les consommateurs eux-mêmes entre l'offre en la matière qui leur est faite et la présence de communautés ethnoculturelles, répondant ainsi, même si de façon floue et partielle, à une question que se pose Santé Canada : en quoi la présence d'immigrants au Canada a-t-elle un impact sur la santé et les pratiques de santé de l'ensemble de la population du pays (Kinnon, 1999)?

8 Les statistiques fournies par l'Enquête Nationale sur la Santé de la Population (ENSP, 1994-95) ne mettent pas en évidence de différence majeure entre la consommation médicamenteuse des immigrants et des non-immigrants (Chen et coll., 1996; Dunn et Dyck, 2000). Au Québec, il semblerait même que les immigrants consomment moins de médicaments (prescrits ou non) que la population de souche (Chamberland, 2002). Ce serait, entre autres, le cas de la communauté chinoise (Pica, 2004). En parallèle, on reconnaît l'existence de pratiques d'automédication, d'inobservance et de pluralisme médical ${ }^{3}$ du côté des immigrants, en particulier, là encore, du côté des communautés asiatiques. Insistant sur ces pratiques comme étant des pratiques à risque, plusieurs études ont ainsi mis en évidence la force de circuits parallèles de distribution de produits non-homologués et d'échanges de substances/produits entre pays d'origine et pays d'accueil (Lam, 1994 ; Wong et coll., 1998 ; Ma, 1999 ; Okumura et coll., 2002). Pour d'autres auteurs, ces pratiques seraient liées à des difficultés d'accès aux services de santé formels et à ces barrières culturelles que l'on a précédemment évoqué, ou encore à des problèmes d'intégration socio-économique (Stephenson, 1995; Jenkins et coll., 1996 ; Groleau, 1998; Ito, 1999, Westberg et Sorensen, 2005 ; Groleau et coll., 2006 ; Ahn et coll., 2006). Quelques auteurs notent en outre que les pratiques asiatiques de pluralisme et d'automédication concerneraient davantage les femmes (Arcury et coll., 
2006 ; Kronenberg et coll., 2006), seraient surtout associées à des problèmes infectieux et chroniques (Wong et coll., 1998) et déterminées par des conceptions de la maladie particulièrement éloignées des valeurs occidentales (Stephenson, 1995; Charupatanapong et coll., 1997 ; Henry, 1999 ; Westberg et Sorensen, 2005) en lien avec une « subculture médicale " populaire importée en terre d'accueil.

9 Pour autant, ces quelques études touchant au phénomène de pluralisme médicamenteux parlent essentiellement de "complémentarité thérapeutique " et englobent l'ensemble des pratiques alternatives (acupuncture, chiropractie, massothérapie, homéopathie, médecines dites traditionnelles, etc.). Elles ne traitent donc pas spécifiquement des médicaments. Ces analyses ne s'interrogent pas véritablement non plus sur la diversité et la variabilité des associations médicamenteuses que ce genre de pratique pluraliste induit: emploi concomitant ou successif (entraînant parfois plus d'une alternance au cours d'une même expérience de maladie) ; variations des formes d'association selon la pathologie (gravité, symptômes, durée, etc.), selon l'interprétation qui est faite de la maladie ou encore en relation avec des représentations individuelles variées, que ce soit du corps, de la douleur ou du médicament, représentations pourtant de plus en plus reconnues pour influer sur les pratiques de santé de la population canadienne dans son ensemble (ICIS, 2000).

\section{Le choix de la population d'étude : Les Vietnamiens de Montréal}

\section{Les Vietnamiens du Canada}

10 Alors que les études en sciences sociales concernant les Vietnamiens du Canada se sont longtemps concentrées sur les réfugiés boat-people et les caractéristiques de leur adaptation sociale (Lanphier, 1979 ; Deschamps, 1985 ; Stephenson, 1995), dont la santé mentale (Beiser, 1988; Beiser et Hyman, 1997; Kirmayer, 2001), avant de s'élargir à l'ensemble de la communauté et de ses pratiques sociales (Nagata, 1987 ; Dorais, 1993), il s'avère que l'on n'a pas de données véritablement probantes sur le profil social et d'abord sociodémographique de cette population. On peut au moins noter son poids statistique non négligeable : 151410 immigrants reçus d'origine vietnamienne ${ }^{4}$ vivaient au Canada en 2001 (Statistique Canada, 2001).

11 Cette communauté serait en fait le produit d'au moins quatre vagues successives d'immigration: une première vague dans les années 1950-1960, faite d'étudiants originaires du Sud Viêt-Nam surtout, partis au lendemain de la guerre d'Indochine (1954) et dans le cadre du plan Colombo5. Estimés à 1500 en 1974, ils vont pour les trois quarts d'entre eux s'installer au Québec, francophone (Dorais, 2000, 2005). Une deuxième vague intervient tout juste au lendemain de la chute de Saigon (1975-1976) et concerne aux environs de 6000 personnes (Indra, 1987; Pfeifer, 1999) issues de l'élite du Sud Viêt-Nam fuyant un pays promis à réunification sous la tutelle communiste (Lâm, 1999). Cette vague est constituée de réfugiés mais aussi de personnes parrainées par des membres de leur famille arrivés lors de la précédente vague. Une troisième vague, la plus importante numériquement, démarre en 1978, à une époque de grandes tensions entre le Viêt-Nam et ses voisins (Chine et Cambodge). Faite de réfugiés qui ont fui précipitamment, par la mer et dans les pires conditions (boat people), elle compte des Vietnamiens mais également des Chinois du Viêt-Nam. Une quatrième vague débutera à 
la fin de 1980 alors que l'ONU est intervenue pour que le Viêt-Nam consente à des départs légaux, et par voie aérienne, dans le respect de principes humanitaires. Entre 1979 et 1982, le Canada aurait, quoiqu'il en soit accueilli près de 58000 réfugiés du ViêtNam (Dorais, 2005). D'un point de vue socio-économique, les vagues 3 et 4 étaient constituées à $80 \%$ de personnes issues des classes populaires ou moyennes (ouvriers, pêcheurs, paysans, petits commerçants, instituteurs, fonctionnaires). Les derniers arrivés étaient en outre tous originaires du Nord du Viêt-Nam et sont passés par des camps de réfugiés (Lâm, 1999). Cette dernière vague prendra fin avec les années $1990^{6}$, la fermeture des camps de réfugiés, l'ouverture du Viêt-Nam à l'économie de marché et le rétablissement de ses relations extérieures.

Le recensement de 2001 établit à 25605 le nombre de personnes d'origine vietnamienne vivant dans la région métropolitaine de Montréal, ce qui place la communauté en cinquième position sur la liste des communautés ethnoculturelles représentées dans la région et fait de la communauté vietnamienne montréalaise la deuxième en importance au Canada après celle de Toronto. Ce recensement ainsi que quelques recherches qualitatives plus ciblées (Pfeifer, 1999 ; Richard, 2003 ; Dorais, 2005) précisent que la communauté vietnamienne de Montréal serait globalement moins favorisée sur le plan socio-économique que la population de souche (10 \% de chômeurs chez les Vietnamiens contre $6 \%$ dans le reste de la population montréalaise) ou que son alter ego torontoise (Lâm, 1999). Selon une étude récente de Renaud et coll. (2003), la communauté vietnamienne de Montréal connaîtrait même un vrai problème d'insertion socioéconomique ${ }^{6}$. Un problème d'insertion qui semble jurer avec le fait que $28,3 \%$ des Vietnamiens de Montréal possèdent un diplôme universitaire contre 14,5\% pour l'ensemble des Montréalais (Statistique Canada, 2001). On sait aussi que la communauté vietnamienne a une forte tradition de valorisation des professions de santé et que lors de la chute de Saigon en 1975, 55 \% du corps médical a fui le pays (Lâm, 1999). Encore aujourd'hui d'ailleurs, même s'il semble que le taux de reproduction socioprofessionnelle baisse au sein de la jeune génération (Blanc, 2006), 10,4\% de la population active vietnamienne de Montréal se trouveraient dans le secteur de la santé contre $5,5 \%$ de la population active de souche (Statistique Canada, 2003).

\section{Histoire et culture médicale vietnamiennes}

13 Si notre choix de la communauté vietnamienne repose donc à la fois sur son poids démographique, sur sa diversité géographique, socio-économique, professionnelle et sur son histoire migratoire, il s'affirme encore davantage au regard des spécificités de la culture médicale vietnamienne. Carrefour des civilisations chinoise et indienne, confucéenne et bouddhique, le Viêt-Nam est doté d'un passé médical marqué par une lente mais dynamique construction d'une médecine nationale reposant sur des pharmacopées locales d'abord influencées par la proximité de la Chine puis nourries par un très riche réservoir pharmacologique régional que les années de revendication de l'indépendance nationale, de guerres et de pénuries (1945-1975) allaient mettre en valeur (Do, 1987 ; Hoang et coll., 1993 ; Wahlberg, 2006).

Dans ce cadre général, on opère généralement une distinction entre " médicament du nord » (thu ố c bắc) et " médicament du sud » (thu ốc nam). Les thu ố c b ắc reposent essentiellement sur des ingrédients contenus dans la pharmacopée traditionnelle chinoise. Qu'il s'agisse de substances issues du règne végétal, animal ou minéral, on les 
retrouve chez les herboristes des communautés chinoise et vietnamienne du Canada ; elles sont généralement proposées sous forme sèche et portent des noms (chinois) savants. Puisant dans la biodiversité plus proprement vietnamienne, les substances qui composent les thu ố c nam sont généralement fournies à l'état brut, frais, et portent un nom vietnamien. La plupart de ces remèdes se préparent en décoction ou tisane ${ }^{7}$. Que ce soit dans la tradition chinoise ou vietnamienne, le médicament peut être préparé (et prescrit) soit par un thérapeute, un « maître de médecine " (th ầ y thu ốc), soit par celui qui fait office de vendeur. Les substances qui le composeront peuvent aussi simplement être achetées par le consommateur, sans intermédiaire, en particulier lorsqu'il s'agit de produits entrant dans la composition de remèdes populaires couramment utilisés. En fait, d'obédience chinoise ou vietnamienne, les pharmacopées auxquelles les Vietnamiens ont recours, sans forcément passer par un médecin ou une prescription, se ressemblent sur bien des points: c'est le mode de préparation, les indications thérapeutiques ou la posologie qui diffèrent éventuellement ${ }^{8}$.

15 À cette vigueur des médecines dites traditionnelles dans la culture vietnamienne s'ajoute une double valorisation historique du recours au médicament comme outil prioritaire de réponse à un problème de santé (Wolffers, 1995; Craig, 2002) et de pratiques de syncrétisme médical. Si on retrouve cette tendance au syncrétisme ailleurs en Asie orientale (Monnais, 2007), dans le cas précis du Viêt-Nam elle a été remodelée avec l'introduction et l'imposition de la médecine moderne dans le cadre de la colonisation française à partir du début du XXe siècle (Monnais, 1999), puis dans celui d'un système de santé national intégratif dès l'avènement de la République Démocratique du Vietnam en 1945, autour d'un accès égalitaire à la médecine occidentale et vietnamienne rendu possible, entre autres, par une politique d'autosuffisance en matière de production et de distribution de médicaments (Wahlberg, 2006) ${ }^{9}$. Le système actuel de santé vietnamien valorise non seulement un recours conjoint à la médecine traditionnelle et à la biomédecine mais aussi à une automédication éclairée. Celle-ci passant, entre autres, par une information médicale ciblée, via essentiellement la presse écrite populaire et de vulgarisation médicale (Finer, 1999; Woodall et coll., 2006), et par le pharmacien encore très souvent prescripteur de médicaments (Craig, 2002).

16 Au-delà de cette valorisation globale, on retrouve souvent l'idée que l'efficacité du médicament au sens large, dans une perspective holistique de la santé, est perçue par les Vietnamiens comme reposant sur sa faculté à rééquilibrer l'harmonie entre corps et esprit, à réparer un dysfonctionnement. Se serait alors construite une vision culturalisée du produit pharmaceutique occidental : trop rapide dans son action, trop fort, éventuellement toxique (Hoang et coll., 1993). Ce produit, chimique de surcroît, serait perçu comme inadapté aux constitutions vietnamiennes, signifiant l'importance du lien entre conception du corps et conception, voire usage, du médicament. Cette lecture du médicament occidental est d'ailleurs dans une certaine mesure valable aussi pour les médicaments chinois considérés comme inadaptés à l'environnement (pathologique, climatique surtout) vietnamien. À l'inverse, la pharmacopée vietnamienne aurait l'avantage de soigner plus en douceur mais aussi plus en profondeur la maladie et ce sans effet secondaire. Lenteur dans l'action qui serait en même temps une des raisons pour lesquelles les Vietnamiens auraient eu de plus en plus recours aux produits pharmaceutiques occidentaux, en particulier pour des maladies aiguës, à partir des années 1930 (Monnais et Tousignant, 2006). Il apparaît en tout cas évident que les représentations vietnamiennes du médicament, 
représentations dynamiques et perméables, se sont complexifiées au fil du temps, puisant dans des traditions profondément ancrées et une histoire collective faite de rencontres culturelles à l'impact profond.

Les pratiques médicamenteuses au Viêt-Nam ont été relativement bien étudiées ces dernières années. Les recherches mettent en évidence, dans un contexte de privatisation du système de santé (1989), lourd de conséquences, des pratiques à risques aux conséquences importantes pour la santé de la population: surconsommation d'antibiotiques et inobservance des prescriptions dont l'effet est de développer des phénomènes de résistance alarmants (Halfvarsson et coll., 2000; Larsson, 2003). Elles signalent aussi que ces pratiques dépendent du revenu (Chang et Trivedi, 2003), du lieu de résidence (urbain vs rural : Halfvarsson et coll., 2000 ; Craig, 2002), de l'âge (Van Duong et coll., 1997), du sexe (Halfvarsson et coll., 2000 ; Okumura et coll., 2002), du type de pathologie concerné ou encore de la durée et de la sévérité du malaise (Van Duong et coll., 1997), ainsi que des logiques profanes, propres à chacun, (Craig, 2000).

\section{Objectifs et hypothèses de recherche}

18 L'objectif principal de notre recherche sur la santé des immigrants vietnamiens, menée avec le soutien des Instituts de Recherche en Santé du Canada (IRSC, Ottawa, 2001-06), était d'aboutir à une meilleure connaissance des pratiques de consommation médicamenteuse de cette communauté dans un environnement culturellement métissé, le Grand Montréal (Montréal et sa banlieue) où l'offre de soins est particulièrement dense. Nous voulions plus spécifiquement identifier et analyser les différentes formes de pluralisme thérapeutique ayant cours auprès de cette population et les déterminants influençant ces pratiques. Nous cherchions en particulier à comprendre l'influence de la culture médicale pré-migratoire individuelle et collective ainsi que le rôle de l'expérience d'immigration selon la durée et le type d'émigration.

19 On peut supposer que les immigrants vietnamiens transposent, du moins en partie, des pratiques thérapeutiques menant à des pratiques de pluralisme thérapeutique denses et complexes, ce qu'une récente étude a d'ailleurs suggéré pour la communauté vietnamienne installée aux États-Unis (Ahn et coll., 2006). Sous-tendant cette hypothèse, nous voulions établir que ces pratiques pluralistes ne sont pas forcément (ou seulement) liées aux problèmes d'adaptation, et d'abord de difficultés d'accès aux services de santé conventionnels, de «barrières culturelles », mais sont aussi le produit de représentations et d'expériences antérieures de la maladie et du soin. Notre dernière hypothèse considérait que les formes de pluralisme thérapeutique mises en avant dans les parcours thérapeutiques des Vietnamiens de Montréal allaient varier selon un certain nombre d'autres déterminants sociaux ainsi qu'en fonction du temps et du type d'immigration.

Dans le présent article, nous nous centrons sur une qualification précise du pluralisme thérapeutique de la communauté étudiée et sur une analyse de la variation de ses formes en fonction des individus interrogés et de leurs représentations (en particulier de la maladie, du soin, de la douleur ou, bien sûr, du médicament). 


\section{Méthodologie}

21 Pour analyser ces pratiques de consommation médicamenteuse, nous avons opté pour une démarche qualitative selon une approche socio-anthropologique de type constructiviste. Cette approche permet de mettre en évidence un large éventail de déterminants des comportements de santé interdépendants (Massé, 1995; Groleau, 1998 ; Gravel et Battaglini, 2000). Il s'agit, entre autres, de rendre compte du caractère dynamique de ces pratiques en lien avec l'expérience d'immigration et le processus d'adaptation au pays d'accueil. Dans cette optique, on considère que l'individu est décideur de ce qu'il veut pour sa santé, qu'il adapte sa culture originelle et son processus de recherche d'aide à l'environnement physique, social et culturel et au système de santé du pays d'accueil. Si la dimension culturelle a donc un rôle pivot dans ces comportements, ce rôle se trouve modulé par d'autres déterminants.

Aux fins de notre analyse, nous avons constitué un échantillon par contraste à la fois révélateur de la population à l'étude et de l'hétérogénéité des facteurs intervenant potentiellement dans sa santé et ses comportements de santé (Pelto et Pelto, 1997). Reposant sur le principe de la diversification externe, la représentativité, sociologique et non statistique, est assurée dans ce type d'échantillon par la diversité des cas et permet de dégager une variété de profil et de stratégies de consommation de médicaments (Pirès, 1997). Nous avons d'abord sélectionné des ménages composés de deux personnes au moins (couple/noyau familial) issus de l'ethnie majoritaire việt ou $k_{i ́ n h}{ }^{10}$ - la diversité ethnique du Viêt-Nam entraînant potentiellement des comportements de santé différenciés (Monnais, 1999). Les familles devaient être installées au Canada depuis plus d'un an, pour éviter de mal interpréter des réalités liées à une période de transition (Wen et coll., 1996), et composées de citoyens ou résidents permanents ( $99 \%$ de la population vietnamienne canadienne en $2001^{11}$ dont $70,9 \%$ nés au Viêt-Nam : MICC, 2005).

23 Les répondants clé devaient être des chefs de famille des ménages retenus. Il s'est avéré que ce sont surtout les maîtresses de maison et/ou le plus âgé de la famille qui ont accepté de nous répondre : c'est pourquoi nous avons une surreprésentation de femmes $(12 / 16)$ et de personnes de plus de 65 ans (5/16). On connait toutefois le rôle central de la femme vietnamienne dans la gestion de la santé familiale ainsi que celui des aînés (Groleau, 1998; Stephenson, 1995). Par ailleurs, plusieurs études récentes ont montré que les femmes et les aînés ont plus tendance que le reste de la population à consommer des médicaments, à avoir recours aux MAC et ont des pratiques de santé pluralistes (Singh et Levine, 2006) surtout s'il s'agit d'immigrants asiatiques (Arcury et coll., 2006; Ahn et coll., 2006). En conséquence, tout en constituant un double biais méthodologique, cette surreprésentation des femmes âgées peut constituer un avantage informatif.

Les critères de diversification de notre échantillon ont été définis en fonction de deux variables stratégiques reconnues pour leur impact sur la santé et les pratiques de santé des immigrants: le statut socio-économique (favorisé vs défavorisé) et le temps d'immigration (ancien vs récent). Alors que certains répondants étaient réticents à indiquer leur revenu, leur statut socio-économique a été établi en fonction du niveau socio-économique du quartier de résidence, ainsi que du niveau d'éducation et de la situation professionnelle à Montréal. Pour ce qui est du temps d'immigration, nous avons réparti notre échantillon en immigrants arrivés avant 1986 et immigrants arrivés 
après $1990^{12}$. Il s'agissait là de prendre en considération une rupture temporelle au Viêt-Nam : le Đố $i$ Mới, ou ère du renouveau, enclenchée en 1986 et marquée par la volonté du Parti Communiste au pouvoir d'ouvrir le pays à l'économie de marché - ce qui se traduira, entre autres, par la privatisation du système de santé vietnamien. Dans ce nouveau cadre politique, l'immigration vietnamienne au Canada allait être une immigration essentiellement économique. Par croisement, ces variables nous ont amenées à constituer quatre types distincts de répondants (immigrants anciens/ favorisés, anciens/défavorisés, récents/défavorisés et récents/favorisés) pour un total de 16 ménages. Si nous avions initialement l'intention de respecter une distribution égale entre ces quatre types, le type favorisé/récent s'est trouvé impossible à recruter. Ce qui s'avère explicable alors que les immigrants les plus récents, issus de milieux plutôt défavorisés, se retrouvent rarement dans une situation socio-économique favorable au Canada (Renaud et coll., 2003).

Nous avons ensuite procédé à des entretiens semi directifs auprès des répondants-clé d'une durée moyenne d'une heure et demi à deux heures. Ces entretiens se sont déroulés entre janvier et novembre 2002, dans la langue choisie par le répondant (vietnamien ou français). Ils ont eu lieu soit au domicile familial soit dans un endroit choisi en accord avec le répondant (siège d'une association communautaire, lieu public, pagode, etc.). Chaque entretien a eu pour principal objectif de rendre explicites les itinéraires thérapeutiques du répondant-clé et éventuellement des différents membres de sa famille, d'identifier en particulier les différents médicaments auxquels chacun faisait appel et dans quel(s) but (s) précis. Pour ce faire, les entrevues ont été balisées par des questions ouvertes: conceptions associées par le répondant au médicament, « traditionnel » et «occidental »; principales expériences pathologiques du répondant et de sa famille au Canada; comportements de recherche d'aide associés à ces expériences, en particulier recours au secteur de soins alternatifs (identification de thérapeutes consultés, des fournisseurs de produits traditionnels); rôle du réseau social dans ces choix ; suivi des traitements consécutifs ; perceptions des différences en matière de prise en charge de la santé entre le Viêt-Nam et le Canada ${ }^{13}$.

Les entretiens ont ensuite été codés à l'aide du logiciel QSR Nvivo. Ce logiciel facilite l'analyse qualitative dans le sens où il permet un découpage rapide de l'information et la reconstruction d'un corpus autour d'un certain nombre de catégories créées en fonction de nos questions de recherche: les savoirs et représentations issus de la culture d'origine, les représentations du médicament, les pratiques de consommation médicamenteuse (pluralisme, automédication, inobservance) ou l'accessibilité aux soins de santé. L'analyse a également consisté en l'établissement d'un répertoire des différents médicaments, produits de santé et substances thérapeutiques évoqués par les répondants lors des entretiens ou repérés dans les pharmacies domestiques que les répondants, pour ceux interviewés à leur domicile, ont bien voulu nous montrer. Pour chaque médicament et produit, nous avons établi sa provenance (géographique, industrielle) et ses indications thérapeutiques usuelles telles que déclarées par les usagers eux-mêmes ${ }^{14}$. L'ensemble des informations mises en relation, les entretiens ont ensuite fait l'objet d'une seconde analyse pour mieux identifier les logiques soustendant les itinéraires thérapeutiques individuels et, en particulier, les facteurs intervenant dans les différentes formes de complémentarité dans le recours aux médicaments : culture originelle, expérience d'immigration, expériences pathologiques 
et des services de santé, réseau familial et social, accessibilité aux services, niveau socio-économique ${ }^{15}$.

\section{Résultats}

\section{Une typologie des médicaments consommés}

Notre première phase d'analyse nous a permis d'établir une liste qualitative des médicaments consommés par nos répondants et de répartir ces derniers en cinq catégories distinctes ${ }^{16}$ :

- Type 1. Des produits traditionnels non manufacturés (thu ốcb ắc et thu ố c nam) achetés sous forme de plantes fraîches ou séchées, vendus par certains commerces asiatiques de Montréal (herboristeries implantées principalement dans le quartier chinois de la ville) ou ramenés directement du Viêt-Nam par un ami ou un parent. 14 sur 16 de nos répondants ont déclaré avoir fait usage de ces produits à un moment de leur vie, surtout dans l'enfance ou au moment des grossesses pour les femmes. 11 ont déclaré en prendre depuis qu'ils sont installés au Canada et pour certains même ont ajouté en consommer davantage depuis leur immigration. Par contre aucun de ces produits ne se retrouve dans les pharmacies domestiques observées ${ }^{17}$.

- Type 2. Des produits «néo-traditionnels » manufacturés provenant généralement de la pharmacopée chinoise ou vietnamienne (thu ố c b ă c et thu ố c nam), fabriqués en Asie ou au Canada, et eux aussi obtenus pour la plupart dans les commerces de la communauté vietnamienne ou chinoise de Montréal. 7 de nos répondants ont déclaré faire usage de ce genre de produits. On notera cependant que lorsqu'on examine le contenu des pharmacies domestiques, ces produits en occupent plus de la moitié et le plus souvent chez ceux qui n'ont pas déclaré en faire un usage régulier (3 répondants sur 7).

- Type 3. Des PSN, non issus des pharmacopées vietnamienne ou chinoise, manufacturés et en vente libre ${ }^{18}$. Cette catégorie inclut des substances telles l'échinacée ${ }^{19}$ ou l'ail, ainsi que des vitamines, des minéraux, oligo-éléments, et des produits homéopathiques. 9 de nos répondants ont déclaré faire usage de ce genre de produits. Dans les pharmacies domestiques, les médicaments de type 3 apparaissent toutefois sous-représentés ( 1 à 2 produits en moyenne), surtout par rapport aux types 2 ou 4.

- Type 4. Des médicaments en vente libre ou OTC (over the counter), généralement nonprescrits et constituant des médicaments de consommation courante (maux de tête, toux, rhume, fièvre, acidité gastrique, etc.). 8 de nos répondants ont déclaré faire usage de ces produits. Lorsqu'on inventorie les pharmacies domestiques, ces produits apparaissent d'ailleurs presque aussi nombreux que ceux de type 2 .

- Type 5. Des médicaments obtenus sur prescription incluant des antibiotiques, antihypertenseurs, anti-cholestérol, anti-inflammatoires et hormones. 13 de nos répondants ont déclaré consommer ce type de médicaments pour lesquels ils disposaient effectivement d'une prescription. Ces médicaments s'avèrent présents dans les pharmacies domestiques en moins grande quantité que les quatre autres types. Par ailleurs, ils ne sont généralement pas rangés dans les mêmes endroits, davantage à portée de main. Plusieurs répondants ont précisé les avoir ainsi plus facilement à disposition sans pour autant les consommer.

Au-delà du fait que cette catégorisation marque la variété des types de produits auxquels nos répondants déclarent avoir eu recours, elle révèle la réalité et la complexité de leurs pratiques pluralistes. Bien sûr, l'accumulation de médicaments 
dans les armoires à pharmacie ne signifie pas qu'ils sont tous consommés. Certains répondants ont d'ailleurs précisé qu'ils gardaient des produits périmés, parfois depuis plusieurs années, simplement pour se souvenir de leur nom. À propos d'un médicament homéopathique contre les effets du décalage horaire, M. Jade, (50 ans, favorisé/ancien) déclare : « ... c'est ça, c'est très bon. C'est très efficace. Il faut prendre toutes les heures quand on est dans l'avion, une pilule à chaque heure. Ça je ne m'en sers plus mais je garde juste prochaine voyage, je peux acheter d'autres même marque ». Reste que tous les répondants ont évoqué des pratiques pluralistes passées ou en cours et 14 sur 16 ont déclaré avoir consommé au cours de leur vie trois au moins des cinq catégories que l'on a identifiées.

\section{Des itinéraires pluralistes complexes}

29 Si on analyse maintenant la consommation individuelle de médicaments selon les problèmes de santé rencontrés, on remarque en fonction du problème identifié - et éventuellement diagnostiqué - que le taux de complexité de la pratique pluraliste varie, i.e. qu'elle inclut plus ou moins de types différents de médicaments. La forme que prend ce pluralisme (recours successif ou simultané dans le temps) diffère également.

On remarque d'abord que pour les maux courants et bénins, les formes de pluralisme sont les plus diversifiées. Par exemple, on note que pour la toux, nos répondants puisent dans les cinq types de médicaments. Pour les problèmes digestifs ou encore les douleurs, qu'il s'agisse de simples maux de tête ou de douleurs d'origine rhumatismale, ils vont utiliser quatre des cinq types mais excluent systématiquement le recours aux plantes sous leur forme brute (Type 1). Pour le cholestérol ou l'hypertension par contre, ils n'auraient recours qu'à deux types de médicaments, les plantes (Type 1) et les médicaments occidentaux prescrits (Type 5). Quant au diabète et aux maladies cardiovasculaires, les répondants semblent suivre la prescription médicale qui leur a été proposée et ne chercher de complément ou d'alternative que rarement. Il apparaitt que ces maladies sont en fait considérées comme particulièrement grave, une gravité perçue qui aurait un impact direct sur l'observance au traitement prescrit. Les allergies sont traitées avec les types 3-4-5 de médicaments, avec une préférence donnée aux anti-histaminiques avec ou sans prescription (en particulier Claritin ${ }^{\circledast}$ ).

31 La consommation de médicaments de nos répondants ne se fait pas seulement en réaction à la présence de maladie ou de symptôme mais aussi en prévention, dans un objectif de maintien global, voire de promotion de la bonne santé physique et mentale. On a pu en particulier observer des pratiques de recours à un ensemble de substances et de médicaments utilisés comme des "panacées préventives». Celles-ci seraient consommées quand la personne le juge utile et en fonction des sensations du corps, et de sa connaissance de ce qui peut être bon pour elle et son maintien en forme. Il s'agit essentiellement de plantes (ginseng, gingembre: type 1) et de médicaments traditionnels manufacturés (Guilin ou Sanjin Watermelon Frost, Qing Re Xiao Yan Ning : type 2) auxquels semblent s'être ajoutées les multi vitamines (type 3). Plusieurs de nos répondants (6), surtout parmi les plus âgés, ont également insisté sur l'importance de l'exercice physique pour maintenir ou améliorer l'harmonie entre corps et esprit (activité physique, marche, qi gong, tai chi, méditation, prière, etc.) ainsi que dans un objectif de prévention de la maladie ou éventuellement de gestion de maladies ou douleurs chroniques. Ainsi, Monsieur Littéraire (61 ans, favorisé/ancien), souffre d'arthrite et d'une tendinite chronique au coude. Après avoir suivi plusieurs 
traitements prescrits et reçu une injection d'hydrocortisone, il s'est orienté vers la pratique du tai chi et du qi gong en plus d'avoir recours à l'acupuncture.

Si on s'intéresse à la façon dont ces produits sont utilisés dans le cadre d'un même itinéraire thérapeutique, on observe plusieurs formes de pluralisme: un pluralisme où l'usage de plusieurs types de médicaments se fait de façon successive, avec des va-etvient entre médicaments de type traditionnel (types 1-2) et médicaments " occidentaux » (types 3-4-5); un pluralisme où l'usage de médicaments occidentaux et traditionnels se fait de façon simultanée. Seules les allergies ne donneraient lieu à aucune association entre médicaments occidentaux et médicaments traditionnels.

\section{Du médicament traditionnel au médicament occidental}

La plupart des répondants qui prennent des médicaments traditionnels, en particulier des thu ố $c b$ á $c$ sous forme de toniques préventifs, semblent décider d'abandonner momentanément leur consommation et s'orienter vers les médicaments de type occidental s'ils jugent que leur santé est en péril ou se détériore. En ce qui concerne la gestion de maladies aiguës (en particulier les infections ORL), plusieurs répondants estiment ainsi que la médecine occidentale peut mieux les traiter que la médecine traditionnelle. Le passage du traditionnel à l'occidental dans la résolution de ce type de problème de santé nous donne par ailleurs à voir un itinéraire en trois temps : recours aux plantes traditionnelles puis aux médicaments vendus sans ordonnance puis, au bout de quelques jours, aux médicaments prescrits, après consultation d'un médecin. Suivant cet itinéraire en trois temps, Mme Sept (53 ans, favorisée/ancienne) consomme une fois par semaine des thu ố $c b$ ác dans une soupe pour rester en bonne santé et bien dormir. Quand elle est enrhumée, elle va de sa propre initiative acheter des médicaments à la pharmacie (elle nous citera même trois produits différents: le Tylénol $\mathrm{l}^{\oplus}, \mathrm{l}^{\prime}$ Atasol ${ }^{\oplus}$ et l'acétaminophène, précisant que sa préférence va à l'Atasol $\left.^{\circledR}\right)$. Mais si après une semaine elle est toujours enrhumée, elle va voir son médecin pour obtenir un traitement susceptible de la guérir.

\section{Du médicament occidental au médicament traditionnel}

L'abandon du médicament occidental au profit d'un ou plusieurs produits de type traditionnel se rencontre dans les itinéraires thérapeutiques de répondants qui ont des représentations très construites du médicament et en particulier du médicament occidental: produit pharmaceutique identifié à une substance chimique, potentiellement toxique, et dont l'ingestion entraînerait des effets secondaires néfastes pour leur corps. Dans la plupart des cas, il s'agit d'individus qui cessent un traitement avant le terme prescrit ou attendent d'avoir fini le traitement prescrit pour se tourner $\mathrm{du}$ côté des produits traditionnels, soit pour faire disparaître les effets secondaires ressentis, soit pour rééquilibrer leur organisme jugé « débalancé », une explication qui a déjà été avancée dans une recherche sur les pratiques de santé de Vietnamiens diabétiques installés aux États-Unis (Mull et coll., 2001). Mme Pâte de Riz par exemple (60 ans, défavorisée/récente) évoque le traitement lourd qu'elle a dû suivre pour un problème pulmonaire - il s'agit en fait de la tuberculose - pendant six mois. Elle a ensuite utilisé des thu ố $c b$ ă $c$ pour rééquilibrer son organisme.

Ce genre d'itinéraire se rencontre par ailleurs surtout chez des personnes qui se sont retrouvées en échec thérapeutique ou ont trouvé «trop lent » l'effet du médicament 
occidental, en particulier lorsqu'il s'agissait de traiter des maladies chroniques pour lesquelles la médecine conventionnelle n'avait apparemment pas de remède miracle - ou quand elle n'avait pas posé de diagnostic probant. On remarquera en outre que ce type d'itinéraire touche fréquemment des problèmes de santé présents dans la nosologie traditionnelle vietnamienne, en particulier les maladies de l'intérieur dues au vent, à l'eau ou au soleil, et les maladies des femmes (Bertrand, 1995 ; Blanc, 1999). Ainsi, Madame Vert (81 ans, défavorisée/ancienne) dit avoir souffert d'un mal au dos qu'elle attribuait au froid. Alors que les médecins n'avaient rien décelé, c'est un traitement sur plusieurs mois à base de thu ố $c b$ á $c$ qui aurait permis, selon elle, que son mal disparaisse. On peut citer aussi le cas de Mme Onze (28 ans, ancienne/ défavorisée) qui a souffert d'une infection vaginale et a décidé d'abandonner le traitement prescrit pour avoir recours à une plante vietnamienne utilisée par la fille d'une amie de sa mère et rapportée directement du Viêt-Nam.

\section{Usages simultanés}

Les itinéraires thérapeutiques dans lesquels les médicaments traditionnels et occidentaux, prescrits ou non, sont utilisés de façon concomitante le sont soit pour en combiner l'effet et surtout l'efficacité perçue sur la maladie ou le symptôme rencontré, soit pour mieux soutenir l'état de santé général et permettre donc une résolution, voire une guérison, plus rapide et dans de meilleures conditions. Ce type d'itinéraire concerne des maux courants et d'abord des douleurs (rhumatismales, à l'estomac) mais également des problèmes de santé chroniques (rhumatismes, hypertension, sinusite chronique).

Prenons l'exemple de M. Mérite (66 ans, ancien/favorisé) : "Quelquefois j'ai m... mal aux muscles là... J'ai pris... Enfin j'applique... j'applique d'abord le Myoflex... Le Myoflex... Le Myoflex, le heavy... le heavy, le pénétrant là! Et puis je fais le... le massage ici, euh... les épaules après je colle Salonpass. Donc si tu veux là, il a... deux types de médicaments là-dessus [rires]... Le baume là, c'est... Myoflex là.... c'est... euh... c'est du type acide salicylique ?... Et puis après on met du Salonpass. ». On peut évoquer à nouveau Mme Pâte de Riz qui, ressentant des douleurs à l'estomac, consomme en même temps un médicament prescrit et des thu ố $c b$ ă $c$. Quant à Mme Excessif (84 ans, défavorisée/ancienne), elle dit prendre sur une base régulière trois médicaments sur prescription pour soigner son hypertension (qu'elle ne considère pas être un problème de santé grave) et ses rhumatismes. Elle déclare aussi avoir peur des effets secondaires et d'ailleurs avoir ressenti des maux de ventre et senti son cœur s'arrêter au bout de trois jours de traitement, mais elle garde sa confiance en un médicament occidental qui selon elle guérit vite. Mais chaque matin, après avoir pris son comprimé pour l'hypertension, elle avale deux compléments alimentaires : un comprimé de vitamine $C$ et une capsule de yaourt. Et chaque après-midi, depuis deux ans, elle boit une décoction de thu ố $c b$ ắc. Elle a aussi recours à l'acupuncture quatre fois par an et pratique le dừ ợng sinh (mélange de tai chi et de qi gong qui constitue une technique de respiration pour régulariser l'énergie). 


\section{Discussion}

\section{Le poids des représentations}

38 L'analyse de notre corpus d'entrevues révèle que les pratiques de santé des membres rencontrés de la communauté vietnamienne incluent fréquemment des pratiques de pluralisme thérapeutique. Nos données montrent surtout l'extrême diversité qualitative de ces pratiques, c'est-à-dire à la fois la variabilité des combinaisons médicamenteuses auxquelles il est fait recours, mais également la complexité des itinéraires thérapeutiques pluralistes possibles pour une seule et même maladie et qui mettent en scène - cas des allergies excepté - des associations de médicaments «traditionnels » (types 1-2) et « occidentaux » (types 3-4-5).

Parmi les facteurs influençant directement ces pratiques que l'on a pu retracer via les itinéraires thérapeutiques identifiés, on doit d'abord noter l'impact des représentations du médicament et des rôles thérapeutiques qui lui sont attribués. Il s'avère très clair que, en contexte d'immigration, le médicament continue pour les Vietnamiens d'être un outil prioritaire de gestion de la maladie mais aussi de prévention et de promotion de la santé. Les regards portés plus spécifiquement sur le médicament occidental sont également concordants avec ceux rencontrés au Viêt-Nam et auprès d'autres communautés vietnamiennes immigrées (Wolffers, 1995; Van Duong et coll., 1997; Mull et coll., 2001 ; Okumura et coll., 2002) : c'est une substance chimique rapidement efficace, en particulier sur certaines maladies graves ou lorsqu'il s'agit de gérer des problèmes qui menacent de durer. Ainsi, évoquant le cas du cancer, Mme Beauté Miracle (26 ans, ancienne/défavorisée) reconnaît des limites à la pharmacopée chinoise : «Mais malgré les herbes chinois euh... il peut pas faire toutes sortes de traitements... comme mettons le cancer, il peut pas traiter, il fait rien sur ça. Tandis que le médicament occidental au moins ça traite. Peut-être que ça traite pas vraiment mais au moins, ça aide. ». Mme Sept et M. Sincère (58 ans favorisé/ancien) ne déclarent eux consommer que des médicaments occidentaux lorsqu'ils sont malades. M. Sincère loue surtout leur rapidité d'action : « ... Moi j'aime bien tous les médicaments plus vite! Guérir seulement. Moi j'aime pas garder, comme les Québécois, la toux là, comme ça. Moi prend tout suite médicament! Guérir tout de suite !... ».

C'est parce que le médicament occidental est vu comme très efficace et rapide dans son action qu'il fait aussi naître le soupçon de ses effets néfastes sur le corps. C'est quand Mme Beauté Miracle commence à ressentir les effets d'un médicament prescrit pour son problème thyroïdien (hormonothérapie), en l'occurrence une fatigue chronique, qu'elle décide de son propre chef d'abandonner le traitement prescrit. Elle ajoute que les herbes chinoises sont certes lentes à agir mais que c'est «sans conséquence " et qu'avec elles, elle va "guérir vraiment». M. Précis (53 ans, ancien/favorisé) nous dit pour sa part que le médicament occidental peut soigner plus vite mais que, en conséquence de son ingestion, on peut ressentir de la chaleur dans son corps, une chaleur qu'il juge négative - en concordance avec certaines représentations traditionnelles connues -, signe potentiel d'aggravation de la maladie. M. Jade exprime aussi ce sentiment paradoxal envers le médicament occidental, à la fois efficace et dangereux pour le corps : «je pense que la médecine euh... actuelle moderne, a sa place, c'est$\grave{a}$-dire que quand on est malade, ça prend des antibiotiques... ça en prend ok! Mais euh, je sais 
que j'en suis plus conscient des produits chimiques comme tel, euh, à long terme, me semble pas être idéal... ».

41 En parallèle, il apparaît clairement que le pluralisme médicamenteux et l'automédication demeurent en terre d'accueil des pratiques de santé jugées positives, voire valorisées renvoyant à des conceptions de la santé et de sa préservation qui insistent sur une prise en charge individuelle responsable que ce soit via le médicament, l'exercice et/ou l'alimentation. Mme Pâte de Riz souffre de rhumatismes et prend beaucoup de thu ố $c b$ ắc pour entretenir sa santé en même temps qu'elle dit observer à la lettre la prescription de son médecin traitement. M. Jade légitime ainsi son penchant pour les PSN et les suppléments alimentaires : il dit croire fermement qu'ils sont bons pour sa santé parce qu'ils lui rappellent des pratiques ancestrales, de son pays natal qu'il juge saines : "[...] alors on, si on peut maintenir notre bonne santé, et guérir certaines maladies mineures... avec des remèdes naturels. C'est... J'aimerais mieux ça comme l'échinacée, les choses comme ça. Les extraits d'ail... les choses comme ça c'est très bon, ça aide et c'est très bon. Certaines alimentations naturelles c'est très bon... on disait, au ViêtNam, de manger du riz brun avec les grains de sésame beaucoup, les choses comme ça ou du tofu... tofu c'est protéines, beaucoup de protéines sans le gras, des choses comme ça... on fait un peu plus attention. »

42 Dans ce genre d'itinéraire, les représentations du corps semblent aussi jouer un rôle primordial dans le choix du médicament et, à nouveau, dans l'attrait (non exclusif) pour les types 1-2-3. De fait, plusieurs répondants insistent sur l'importance d'entretenir son corps, de faire en sorte de le rendre fort pour qu'il soit capable de lutter seul contre la maladie (concept de la natura medicatrix). C'est d'ailleurs dans cette optique que Mme Onze pense qu'il n'est pas toujours nécessaire pour elle de suivre un traitement complet - elle va toutefois s'interroger au préalable sur la gravité de son problème - son corps pouvant, selon elle, se défendre seul. Rappelons ici que si le produit considéré « naturel », qu'il soit à base de plantes, asiatique ou occidental (types 1-2-3), dispose d'une place de choix dans les pratiques de consommation médicamenteuse de nos répondants, il s'agit de produits très rarement prescrits par un médecin. Leur consommation entraîne donc forcément des pratiques d'automédication au sens conventionnel, biomédical du terme.

On note également l'importance des représentations de la maladie, en particulier en ce qui a trait à son caractère chronique ou aigu, sa gravité perçue, les symptômes qu'elle induit, ou encore la construction étiologique profane dont elle est objet. Pour ce qui est de la gestion des douleurs par exemple, qu'il s'agisse de simples maux de tête (aigu) ou des douleurs d'origine rhumatismale (chronique), les immigrants vietnamiens interrogés iraient jusqu'à utiliser quatre des cinq types de médicaments que l'on a identifiés mais excluraient systématiquement la consommation de plantes sous leur forme brute (Type 1). Ce genre de pratique éclatée semble lié à l'urgence perçue avec laquelle le symptôme doit être pris en charge ${ }^{20}$. L'intensité de la douleur, du malaise conduirait par ailleurs à recourir à des médicaments manufacturés exclusivement, ne nécessitant aucune forme de préparation, permettant de gagner du temps (Vuckovic, 1999). La préférence va en outre aux médicaments manufacturés de type occidental jouissant davantage que les autres de cette réputation de rapidité dans l'action (dont sur les symptômes) que l'on a déjà évoquée. M. Aimé (80 ans, ancien/favorisé) n'hésite pas à parler de sa petite réserve, bien utile en cas de douleur : «[...] J'ai des médicaments de réserve hivernales... si je me sens pas bien... je prends l'aspirine ou quelque chose de même et 
si ça s'aggrave, je vais voir le médecin. Si c'est pas grave... par exemple, de temps en temps j'ai mal aux genoux mais pas toujours alors si je me sens mal je vais... prendre du orinol [Tylénol@] c'est pour maux de tête... condensé pour calmer la douleur... je ne prends pas tous les jours... seulement quand ça fait mal...j'ai toujours des médicaments de réserve [...]».

En parallèle, l'hypertension, l'arthrose et les rhumatismes, les problèmes de santé chroniques en général (problèmes cardiaques, diabète et hypercholestérolémie), semblent le plus souvent considérés par nos répondants comme étant des manifestations d'un dysfonctionnement du corps attribué essentiellement au vieillissement. Si cette interprétation renvoie là encore au maintien de représentations traditionnelles de la maladie, elle ne signifie pas pour autant que ceux qui en sont atteints en minimisent la gravité. En conséquence d'ailleurs, les répondants qui ont déclaré souffrir de ces problèmes (7) recourraient majoritairement (4) aux types 1 et 5 en conjugaison. Ils suivraient le traitement prescrit par leur médecin traitant et, simultanément, se tourneraient vers d'autres alternatives pour supporter leur organisme "débalancé " (et soumis en outre à un traitement potentiellement dangereux). Dans ce cadre, Mme Vert dit suivre ses prescriptions pour son hypertension et pour son arthrose ; elle ajoute faire une cure de thu ố $c b$ á $c$ pendant trois mois quand elle se sent tout particulièrement fatiguée. Il nous faut peut-être suggérer ici que les personnes qui déclarent une ou plusieurs maladies chroniques sont en général les plus âgées. En conséquence, elles sont celles qui ont potentiellement la plus large et la plus longue expérience des plantes traditionnelles.

\section{L'immigration comme facteur culturel}

Face à ces éléments d'analyse, il nous semble pertinent de nous intéresser plus particulièrement aux rôles thérapeutiques qui sont précisément dévolus à chaque type de médicament identifié et, par extension, au système médical dont chacun dépend (médecine conventionnelle, traditionnelle chinoise et traditionnelle vietnamienne, alternative et complémentaire) pour ainsi mieux comprendre certains des déterminants culturels du pluralisme médical. Il nous apparaît également indispensable d'approfondir la réflexion sur l'impact spécifique de l'expérience d'immigration dans l'émergence de nouveaux problèmes de santé et de nouvelles réponses à ces problèmes.

Ainsi, on a déjà pu remarquer que les allergies prennent une place importante dans les discours sur les expériences marquantes de maladies en terre d'accueil d'un grand nombre de répondants, en particulier de femmes migrantes anciennes/favorisées. Des problèmes qui sont le plus souvent évoqués comme étant liés à l'environnement et au climat canadien. Si plusieurs recherches sur les allergies respiratoires et l'asthme chez les migrants tendent à montrer que le mode de vie et les facteurs environnementaux dans les pays occidentaux conjugués à la durée de séjour facilitent en effet la survenue de tels troubles (Leung et coll., 1994 ; Rottem et coll., 2005), il n'en reste pas moins que les migrants vietnamiens interrogés reformuleraient en contexte d'immigration un discours sur l'environnement et le climat et opèreraient des glissements étiologiques et pathologiques qui donnent lieu à des pratiques de pluralisme thérapeutique renouvelées. Le recours à l'échinacée (type 3) serait particulièrement révélateur de ce renouvellement des pratiques, jouant un rôle similaire aux types 1 et 2 , permettant de 
rééquilibrer l'organisme plutôt que de jouer sur les symptômes liés au problème allergique.

Dans le même ordre d'idée, la gestion médicamenteuse de la ménopause semble associée au contexte d'immigration, en particulier à un mouvement d'acculturation à des repères occidentaux en la matière mais aussi au vécu du traitement hormonal. On a pu ainsi noter que si les hormones font généralement peur aux femmes lorsqu'elles sont jeunes ${ }^{21}$, enceintes ou susceptibles de l'être, ou encore dans le cas d'un problème thyroïdien («Parce que me dire d'essayer toutes les hormones pour lequel est mieux pour moi là mais là, vu que j'suis jeune là, j'ai pas essayé les hormones... pour pas jouer dans ça... les hormones» expose Mme Beauté Miracle), lorsqu'il s'agit de prendre en charge la ménopause le rapport à ce genre de médicament change. En fait, aucune des répondantes interrogées sur la prise d'hormones dans ce cadre n'a émis de crainte visà-vis du traitement, au contraire : plusieurs se sont dites soulagées de nombreux maux et surtout des migraines qui les assaillaient régulièrement (Mme Rizière, 60 ans ; Mme Mérite, 66 ans, anciennes/favorisées).

Il apparaîtrait enfin que l'offre de soins, en terre d'accueil, en particulier l'offre de MAC et de PSN à Montréal, joue un rôle dans la recomposition, la dynamisation et éventuellement la complexification des pratiques de pluralisme médicamenteux, marquant ainsi à la fois la pérennité culturelle d'une valorisation du pluralisme et de l'automédication mais également une forme claire d'acculturation aux repères en santé de la population de souche via le recours aux médicaments. L'inclusion des vitamines et des suppléments alimentaires (vitamine $C$ et multi vitamines en particulier) dans la prévention de la maladie peut être à cet égard significatif. Les vitamines font en effet partie des PSN qui sont utilisés par les migrants vietnamiens comme une "panacée préventive ", remplaçant sans doute, du moins en partie, la gamme de toniques disponibles au Viêt-Nam et présent dans la pharmacopée traditionnelle vietnamienne (Craig, 2000). Précisons ici qu'il s'agit aussi du PSN le plus consommé au Canada (57\% des Canadiens y auraient régulièrement ou occasionnellement recours: Ipsos Reid, 2005). De la même façon, en cas de réaction allergique, les Vietnamiens et surtout les Vietnamiennes - ce sont les femmes qui évoquent ce genre de problème et le lient au climat canadien - se tourneraient du côté de l'échinacée (deuxième PSN le plus consommé par la population canadienne: Singh et Levine, 2006). Ils précisent qu'ils l'utilisent pour renforcer leur système immunitaire d'une manière transitoire et naturelle, une indication thérapeutique en totale concordance avec celle de l'ephedra sinica que proposent les pharmacopées chinoise et vietnamienne pour ce genre de problème ${ }^{22}$ (Hoang et coll., 1993).

\section{Conclusion}

En identifiant l'éventail des types de médicaments consommés par les individus constituant notre échantillon, nous avons mis en évidence non seulement la réalité, partagée, de pratiques de pluralisme médical au sein de cette communauté mais également la complexité et la variabilité des formes que prend ce pluralisme. $\mathrm{Au}$ travers de la reconstruction d'un certain nombre d'itinéraires thérapeutiques individuels, nous avons également pu identifier un certain nombre de facteurs culturels présents dans les pratiques de santé de la population étudiée. En recourant à l'étude qualitative centrée sur une communauté ethnoculturelle, sur les pratiques 
médicamenteuses, on peut mettre en évidence l'importance d'un mouvement de reconfiguration partielle et dynamique des repères en matière de recours aux soins et notamment au médicament qui dépendrait moins de problèmes d'accessibilité aux services conventionnels ou d'intégration au pays d'accueil que d'un processus dynamique et complexe d'adaptation. Adaptation qui ne fait pas pour autant disparaître un certain nombre de repères étroitement liés à une histoire et à une culture médicale vietnamienne fortes et originales et, bien sûr, aux expériences prémigratoires, en particulier les expériences pathologiques. Plus largement, cette étude marque la complexité du phénomène d'engouement pour les médecines alternatives et complémentaires. Elle interroge la notion de perméabilité entre les systèmes médicaux dans un contexte occidental multiculturel éclairant ainsi les réflexions politiques et professionnelles actuelles autour de la possible construction de systèmes de santé intégratifs dans les pays industrialisés.

\section{BIBLIOGRAPHIE}

AHN Andrew C., NGO-METZGER Quyen, LEGEDZA Anna T.R., MASSAGLI Michael p. , CLARRIDGE Brian R. et PHILLIPS Russell S. (2006) Complementary and alternative medical therapy use among Chinese and Vietnamese Americans : prevalence, associated factors, and effects of patientclinician communication, American Journal of Public Health, 96 (4), pp. 647-653.

ARCURY T., SUERKEN C., GRZYWACZ J., BELL R., LANG W., QUANDT S. (2006) « Complementary and Alternative Medicine Use among Older Adults : Ethnic Variation », Ethnicity \& Disease, 16 : 723-731.

BEISER Morton (1988) Influences of Time, Ethnicity, and Attachment on Depression in Southeast Asian Refugees, American Journal of Psychiatry, 145 (1), pp. 46-51.

BEISER Morton et HYMAN Ilene (1997) Refugees' Time Perspective and Mental Health, American Journal of Psychiatry, 154 (7), pp. 996-1002.

BENOIST Jean (1996) Soigner au pluriel. Essai sur le pluralisme médical, Paris, Éditions Karthala, $520 \mathrm{p}$.

BERTRAND Didier (1995) Représentations concernant les enfants et la maladie chez les sampaniers de la Rivière des Parfums à Huê, Études Vietnamiennes, 116 (2), pp. 27-61.

BIBEAU Gilles, CHAN YIP Alice, LOCK Margaret, ROUSSEAU Cécile (1992) La santé mentale et ses visages : vers un Québec pluriethnique au quotidien Montréal, Gaëtan Morin éditeur, 289 p.

BLANC Marie-Eve (1999) «Des “maladies sociales” à l'épreuve de la modernité ? Aperçu ethnohistorique sur la gestion des épidémies au Vietnam » [ "Social diseases » tried by modernity? Ethno-historical survey on epidemic management in Vietnam], Mekong Malaria Forum, Regional Malaria Control Program in Cambodia, Laos and Vietnam supported by the European Commission in collaboration with ACTMalaria, SEAMEO TROPMED and WHO, n 4, oct. 1999, pp. 55-73.

BLANC Marie-Eve (2006) La difficulté d'échapper à sa « bonne réputation » : l'immigrant vietnamien dans la presse en France et au Canada, Transtext(e) $s$-Transcultures, 1, pp. 123-137. 
CHAMBERLAND C. (2002) «Consommation de médicaments » dans Santé et bien-être, immigrants récents au Québec : une adaptation réciproque? Rapport de l'Étude auprès des communautés culturelles 1998-1999, Québec, Institut de la statistique du Québec, chapitre 14, pp. 271-288.

CHANG F.R. et TRIVEDI p. K. (2003) Economics of self medication : theory and evidence, Health Economics, 12 (9), pp. 721-739.

CHARUPATANAPONG N., CHEUNG H.C., HUYNH J.H., NGUYEN K.V. (1997) Self-medication practices in the low income Asian elderly, Journal of Pharmacoepidemiology, 6, pp. 5-22.

CHEN Jiajiang, NG Edward et WILKINS Russel (1996) La santé des immigrants au Canada en 1994-1995, Rapports sur la santé, 7 (4), pp. 33-45.

CHEVALIER Serge et GRAVEL Sylvie (2002) Utilisation des services de santé et des services sociaux, in Santé et Bien-être, immigrants récents au Québec : une adaptation réciproque ? Étude auprès des communautés culturelles 1998-1999, Québec, Institut de la Statistique, pp. 247-259.

COLLIN Johanne, DOUCET Hubert, LAFORTUNE Denis, MONNAIS Laurence, OTERO Marcelo, BLANC Marie-Eve et PROULX Michelle (2005) Le médicament comme objet social et culturel : Recension des écrits et propositions sur les perspectives et les objets de travail à prioriser, Québec, Conseil de la Santé et du Bien-Etre, $66 \mathrm{p}$.

COLLIN Johanne, OTERO Marcelo et MONNAIS Laurence (2006) Le médicament au cœur de la socialité contemporaine : regards croisés sur un objet complexe, St Foy : Presses de l'Université du Québec, $310 \mathrm{p}$.

CRAIG David (2000) Practical logics : the shapes and lessons of popular medical knowledge and practice - examples from Vietnam and Indigenous Australia, Social Science \& Medicine, 51 (5), pp. 703-711.

CRAIG David (2002) Familiar Medicine. Everyday Health Knowledge and Practice in Today's Vietnam, Honolulu, University of Hawaii Press, 288 p.

DESCHAMPS Gilles (1985) Étude longitudinale sur l'adaptation socio-économique des réfugiés indochinois au Québec : la deuxième année de séjour, Québec, Ministère des Communautés Culturelles et de l'Immigration, cahier $\mathrm{n}^{\circ}$ 3, $122 \mathrm{p}$.

VAN DUONG, D., C.W. BINNS, C.W. et T. VAN LE (1997) Availability of antibiotics as over-thecounter drugs in pharmacies : a threat to public health in Vietnam, Tropical Medicine \& International Health, 2 (12), pp. 1133-1139.

DO Tat Loi (1987) Medicinal Plants and Drugs from Vietnam, Hanoi, Scientific and Technology Publishing House (5e éd), 1274 p.

DORAIS Louis-Jacques (1993) Vie religieuse et adaptation : Les Vietnamiens de Montréal, Culture, 13 (1), pp. 3-16.

DORAIS Louis-Jacques (2000) La recherche sur l'immigration vietnamienne au Canada, Moussons, 1: 93-105.

DORAIS Louis-Jacques (2005) Vietnamese in Canada. In C. Ember, M. Ember, \& I. Skoggard eds., Encyclopedia of Diasporas : Immigrant and Refugee Cultures Around the World, New York : Springer Science \& Business Media, vol. 2, pp. 1149-1158.

DUNN James \& DYCK Isabel (2000) Social Determinants of Health in Canada's Immigrant Population : Results from the National Population Health Survey, Social Science \& Medicine, 51 (4), pp. 1573-1593. 
FINER David (1999) Pressing Priorities : Consumer Drug Information in the Vietnamese Marketplace, Stockholm,IHCAR, Karolinska Institutet.

GLOBERMAN Steven (1998) Immigration and Health Care Utilisation Patterns in Canada, Vancouver, Vancouver Centre of excellence, Research on Immigration and Integration in the Metropolis Working Paper Series, $n^{\circ}$ 98-08, 33 p.

GRAVEL Sylvie et BATTAGLINI Alex (2000) Culture, santé et ethnicité. Vers une santé publique pluraliste, Montréal, Régie régionale de la santé et des services sociaux de Montréal-centre/ Direction de la santé publique de Montréal centre, $243 \mathrm{p}$.

GROLEAU Danielle (1998) Déterminants culturels et l'approche écologique : le cas de la promotion de l'allaitement chez les immigrantes vietnamiennes, Thèse de doctorat, Département de médecine sociale et préventive, Université de Montréal, ix + 189 p.

GROLEAU Danielle, SOULIÈRE Margot \& KIRMAYER Laurence J. (2006) Breatfeeding and the cultural configuration of social space among Vietnamese immigrant woman, Health \& Place, 12, pp. 516-526.

GUBERMAN Nancy et MAHEU Pierre (1997) L'utilisation des ressources d'aide par les personnes âgées chinoises du centre-ville de Montréal, Montréal, CLSC centre-ville, $247 \mathrm{p}$.

HALFVARSSON J. HEIJNE N., LJUNGMAN p. , HAM MN., HOLMGREN G., TOMSON G. (2000) Knowing when but not how ! - mothers' perceptions and use of antibiotics in a rural area of Viet Nam, Tropical Doctor, 30 (1), pp. 6-10.

HENRY Rebecca (1999) Measles, Hmong, and Metaphor : Culture Change and Illness Management under Conditions of Immigration, Medical Anthropology Quarterly, 13 (10), pp. 32-50.

HOANG Bao Chau, PHO Duc Thao et HUU Ngoc (1993) La médecine traditionnelle vietnamienne, Hanoi, The Gioi, 274 p.

HYMAN Ilene (2001) Immigration et Santé, Série document de travail sur les politiques de santé 01-05, Ottawa, Santé Canada, $101 \mathrm{p}$.

HYMAN Ilene \& DUSSAULT Gilles (1991) The Effect of Acculturation on Perinatal Health in Montreal, Santé - Culture - Health, 8 (3), pp. 339-365.

INDRA Doreen M. (1987) Social Science Research on Indochinese Refugees in Canada dans Dwok B. Chan \& Doreen M. Indra eds.Uprooting, Loss, and Adaptation: The Resettlement of Indochinese Refugees in Canada, Ottawa, Canadian Public Health Association.

Institut Canadien d'Information sur la Santé (ICIS/CIHI) (2003) Drugs Expenditure in Canada, 1985-2002, Ottawa : ICIS/CIHI, 117 p.

ITO K. L. (1999) Health culture and the clinical encounter : Vietnamese refugees' responses to preventive drug treatment of inactive tuberculosis, Medical Anthropology Quarterly, 13 (3), pp. 338-364.

JENKINS Christopher, LE Thao, MCPHEE Stephen, STEWART Susan \& HA Ngoc The (1996) Health care access and preventive care among Vietnamese immigrants : do traditional beliefs and practices pose barriers ?, Social Science \& Medicine, 43 (7), pp. 1049-1056.

KINNON Dianne (1999) Recherche sur l'immigration et la santé au Canada : Un aperçu, Ottawa, Santé Canada, $89 \mathrm{p}$.

KIRMAYER Lawrence J. (2001) Cultural Variations in the Clinical Presentation of Depression and Anxiety : Implications for Diagnosis and Treatment, Journal of Clinical Psychiatry, 62 (13), pp. 22-30. 
KRONENBERG F., CUSHMAN L., WADE C., KALMUSS D. \& CHAO M. (2006) Race/Ethnicity and Women's Use of Complementary and Alternative Medicine in the United States : Results of a National Survey, American Journal of Public Health, 96 (7) pp. 1236-1242.

LAM Lawrence (1994) Self-assessment of Health Status of Aged Chinese-Canadians, Journal of Asian and African Studies, 29 (1/2), pp. 77-90.

LAM Thanh Liêm (1999) Reconversion professionnelle et intégration sociale de la communauté vietnamienne au Canada, Églises d'Asie, 279 (Supplément 1/99),

http://eglasie.mepasie.org/1999/janvier/vietnam/1 99/doc6 1/index.htm

LANPHIER Michael (1979) A study of Third-World Immigrants, Discussion paper $n^{\circ} 144$, Economic Council of Canada, Ottawa, $76 \mathrm{p}$.

LARSSON M. (2003) Antibiotic Use and Resistance : Assessing and Improving Utilization and Provision of Antibiotic and Other Drugs in Vietnam, Stockholm, Karolinska University Press.

LEUNG R.C., CARLIN J.B., BURDON J.G., \& CZARNY D. (1994) Asthma, allergy and atopy in Asian immigrants in Melbourne, Medical Journal of Australia, 161 (7), pp. 418-425.

MA Xueqin Grace (1999) Between Two Worlds : The Use of Traditional and Western Health Services by Chinese Immigrants, Journal of Community Health, 24 (6), pp. 421-437.

MASSÉ Raymond (1995) Culture et santé publique. Les contributions de l'anthropologie à la prévention et à la promotion de la santé, Montréal, Gaëtan Morin Editeur, 499 p.

MCDONALD James T. \& KENNEDY Steven (2004) Insights into the « healthy immigrant effect » : health status and health service use of immigrants to Canada, Social Science \& Medicine (sous presse).

Ministère de l'Immigration et des Communautés Culturelles (MICC) (2005) Portrait statistique de la population d'origine ethnique vietnamienne, recensée au Québec en 2001, Québec, Gouvernement du Québec, $10 \mathrm{p}$.

MONNAIS Laurence (1999) Médecine et colonisation. L'aventure indochinoise, 1860-1939, Paris, CNRS Éditions, $489 \mathrm{p}$.

MONNAIS Laurence \& TOUSIGNANT Noemi (2006), The Colonial Life of Pharmaceuticals, Accessibility to Health Care, Consumption of Medicines and Medical Pluralism in French Vietnam, 1905-1945, Journal of Vietnamese Studies, 1 (1-2), pp. 131-168.

MONNAIS Laurence (2007) Medical Traditions in Southeast Asia : From Syncretism to Pluralism, dans William F. Bynum et Helen Bynum dir, Dictionary of Medical Biography, Westport, Ct, Greenwood Publishing Group, vol. 1, pp. 67-78.

MULL D.S., NGUYEN N. \& MULL J.D. (2001) Vietnamese diabetic patients and their physicians : what ethnography can teach us, Western Journal of Medicine, 175, pp. 307-311.

NAGATA Judith (1987) Is multiculturalism sacred ? The power behind the Pulpit in the religious congregations of Southeast Asian Christians in Canada, Canadian Ethnic Studies, 19 (2), pp. 26-43.

NAJM W., REINSCH S., HOEHLER F. \& TOBIS J. (2003) Use of complementary and alternative medicine among the ethnic elderly, Alternative Therapies in Health and Medicine, 9 (3), pp. 50-57.

NGUYEN M.T. \& VU T.H. (2001) The Oral Contraceptive Pill in VietNam : Situation, Client Perspectives and Possibilities for Promotion, Asia-Pacific Population Journal, 16 (4), pp. 31-48.

OKUMURA Junko, WAKAI Susumu \& UMENAI Takusei (2002) Drug utilisation and self-medication in rural communities in Vietnam, Social Science \& Medicine, 54 (12), pp. 1875-1886. 
Organisation Mondiale de la Santé (OMS) (2002) Stratégie de l'OMS pour la Médecine Traditionnelle pour 2002-2005, Genève, OMS, 65 p.

PELTO p. J. \& PELTO G.H. (1997) Studying knowledge, culture, and behaviour in applied medical anthropology, Medical Anthropology Quarterly, 11 (2), pp. 147-163.

PFEIFER Mark E. (1999) «Community», Adaptation and the Vietnamese in Toronto, thèse de doctorat, Toronto, University of Toronto.

PICA L. (2004) Faits saillants de l'Étude auprès des communautés culturelles 1998-1999 sur les immigrants d'origine chinoise, Québec, Institut de la statistique du Québec, 8 p.

PIRES Alvaro (1997) De quelques enjeux épistémologiques d'une méthodologie générale pour les sciences sociales dans Jean Poupart dir, La recherche qualitative. Enjeux épistémologiques et méthodologiques, Boucherville, Gaëtan Morin éditeur, pp. 3-54.

RENAUD Jean, PICHÉ Victor et GODIN Jean-François (2003) L'origine nationale et l'insertion économique des immigrants au cours de leurs dix premières années au Québec, Sociologie \& Sociétés, 35 (1), pp. 165-184.

RICHARD Eric \& DORAIS Louis-Jacques (2003) Statistical Profile of Immigrants of Vietnamese Origin in Quebec and in Canada : Comparison of 1991, 1996 and 2001 Data, Review of Vietnamese Studies, 3-1, $9 \mathrm{p}$.

http://vstudies.20megsfree.com/Dorais\%20and\%20Richard.pdf

ROSSI Ilario (1997) Corps et Chamanisme. Essai sur le pluralisme médical. Paris, Armand Colin, 209 p. ROTTEM M., SZYPER-KRAVITZ M. \& SHOENFELD Y. (2005) Atopy and asthma in migrants, International Archives of Allergy and Immunology, 136 (2), pp. 198-204.

Santé Canada (2003) Loi sur les Aliments et Drogues. Règlement sur les produits de santé naturels, Gazette du Canada Partie II, 137 (13), 5 juin 2003, pp. 1532-1607.

SINGH S. \& LEVINE M. (2006), « Natural Health Product Use in Canada : Analysis of the National Population Health Survey », Canadian Journal of Clinical Pharmacology, 13 (2), pp. 240-250.

Statistique Canada (2001) Enquête Sociale Générale : un aperçu, Ottawa, Statistique Canada, 12 p. Statistique Canada (2003) Recensement de 2001 : série « analyses ». Portrait ethnoculturel du Canada : une mosaïque en évolution, Ottawa, Statistique Canada, 65 p.

Statistique Canada (2005) Étude : les dépenses personnelles en médicaments sur ordonnance, 1992 à 2002, Le Quotidien, 23 septembre 2005, Ottawa : Statistique Canada, p. 2.

Ipsos-Reid (2005) Enquête de référence menée sur les produits de santé naturels auprès des consommateurs. Ottawa, Statistique Canada et Ipsos-Reid, 97 p.

STEPHENSON Peter H. (1995) Vietnamese refugees in Victoria, BC : An Overview of immigrant and refugee health care in a medium-sized Canadian urban center, Social Science \& Medicine, 40 (12), pp. 1631-1642.

VUCKOVIC Nancy (1999) Fast Relief : Buying Time with Medications, Medical Anthropology Quarterly, 13 (1), pp. 51-68.

WAHLBERG Ayo (2006) Bio-politics and the promotion of traditional herbal medicine in Vietnam, Health, 10 (2), pp. 123-147.

WEN Shi Wu, VIVEK Goel \& WILLIAMS J. Ivan (1996) Utilization of health care services by immigrants and other ethnic/cultural groups in Ontario, Ethnicity \& Health, 1 (1), pp. 99-109. 
WESTBERG S., SORENSEN T. (2005), « Pharmacy-Related Health Disparities Experienced by NonEnglish Speaking Patients : Impact of Pharmaceutical Care ", Journal of the American Pharmacists Association, 45 (1), pp. 48-54.

WHYTE Susan R., VAN DER GEEST Sjaak \& HARDON Anne (2002) Social Lives of Medicines, Cambridge, Cambridge University Press, 200 p.

WOLFFERS Ivan (1995) The role of pharmaceuticals in the privatization process in Vietnam's health-care system, Social Science \& Medicine, 41 (9), pp. 1325-1332.

WONG Leo Kwan-Ho, JUE Perpetua, LAM Augustine, YEUNG William, WAH Yuen Cham \& BIRTWHISTLE Richard (1998) Chinese herbal medicine and acupuncture -How do patients who consult family physicians use these therapies, Canadian Family Physician, 44, pp. 1009-1015.

WOODALL Erica D., TAYLOR Victoria M., YASUI Yutaka, NGO-METZGER Quyen, BURKE Nancy, THAI Hue \& JACKSON J. Carey (2006) Sources of Health Information Among Vietnamese American Men, Journal of Immigrant and Minority Health, 8 (3), pp. 263-272.

\section{NOTES}

1. Linguistiques et religieuses essentiellement (Stephenson, 1995 ; Guberman et coll., 1997).

2. Cette nouvelle loi tente de structurer et de surveiller à la fois la production, la distribution et la consommation des PSN, obligeant désormais les producteurs et les distributeurs à garantir la qualité de ces produits et à obtenir une licence d'exploitation sur le modèle de celle réclamée pour les bio-médicaments (Santé Canada, 2003).

3. La notion de pluralisme médical a été définie et utilisée par les anthropologues (Benoist, 1996 ; Rossi, 1997 ; Whyte et coll., 2002) avant d'intéresser la santé publique et les gestionnaires de la santé. L'étude de ce phénomène se concentre sur l'analyse de la coexistence de plusieurs systèmes médicaux et traditions de soins au sein d'une même société ou d'une même communauté culturelle, en particulier au travers de l'examen des pratiques de santé des individus.

4. D’origine vietnamienne dans le sens de «né(e) au Viêt-Nam ». Nous ne disposons en effet pas de données sur l'origine ethnique (Viêt, chinoise ou autre) de cette communauté.

5. En janvier 1951, à Colombo (Sri Lanka), le Canada, et six de ses partenaires du Commonwealth mettaient sur pied un programme d'aide étrangère. Le Plan Colombo est devenu un cadre de coopération internationale afin d'aider les pays à relever leur niveau de vie avant d'être l'origine $\mathrm{du}$ programme d'aide extérieure du Canada (Agence Canadienne de Développement International, ACDI).

6. $76 \%$ des migrants d'origine vietnamienne vivant au Québec sont arrivés au Canada avant 1990 contre $24 \%$ après 1990 marquant d'ailleurs un vieillissement de la communauté comparée à d'autres communautés ethnoculturelles de la province et du Canada (Ministère de l'Immigration et de Citoyenneté Canada, MICC, 2005).

7. C'est pourquoi d'ailleurs on dit toujours « boire un médicament » en vietnamien.

8. Par exemple, vó quýt est un thuốc nam, mais trần bì (mot sino-vietnamien) est un thuốc bắc pourtant il s'agit de la même chose de l'écorce de mandarine. Ou encore cây quếqui est un thuốc nam et quế khô qui est un thuốc b ắc. Khô signifie sec, c'est seulement l'état de la plante qui le fait changer de pharmacopée.

9. L'OMS faisait encore récemment référence à ce système sanitaire, élargi à l'ensemble du ViêtNam après la réunification de 1975, comme à un modèle dans son programme de promotion des systèmes de santé intégratifs (OMS, 2002).

10. Việt et kính sont deux ethnonymes qui désignent l'ethnie majoritaire du Viêt-Nam. 
11. Il est important de préciser ici que résidents permanents et citoyens bénéficient des mêmes avantages en matière de santé et de soins, c'est-à-dire un accès gratuit aux services.

12. La période de 1986 à 1990 n'est pas couverte pour respecter le principe de contraste.

13. Précisons que les personnes interviewées ont choisi un pseudonyme ou bien s'en sont vu attribuer un pour conserver leur anonymat au regard de conditions de santé particulières et de pratiques de santé qui pourraient les placer dans des positions stigmatisantes. Ce sont ces pseudonymes qui sont utilisés ici.

14. Dans la mesure du possible, nous avons essayé d'identifier l'origine précise de chaque produit. Sans en faire un objectif en soi ce répertoire pouvait nous permettre de mieux qualifier les pratiques de pluralisme thérapeutique.

15. Cette seconde étape dans l'analyse fera l'objet de publications ultérieures.

16. Ces cinq catégories constituent une construction a posteriori. Cela signifie qu'elles n'ont pas été identifiées ainsi par nos répondants même si ces derniers ont un discours construit sur le médicament qui distingue en particulier le prescrit du non prescrit, le «nôtre " de «l'occidental », le chimique du naturel.

17. On émet ici l'hypothèse que cette absence est en partie dû à une question de conservation: ces produits doivent être préparés et consommés rapidement à la différence des autres types de produits, tous manufacturés.

18. Du moins au moment où les entrevues ont été faites, avant l'entrée en vigueur de la loi de janvier 2004. Précisons ici que certains médicaments traditionnels manufacturés pourraient être considérés comme des PSN canadiens, ayant obtenu un DIN (Drug Identification Number) au Canada et étant vendu dans les pharmacies. C'est le cas du sirop de Mme Pearl ou du fameux Baume du Tigre. Le DIN est au Canada ce que l'AMM (Autorisation de Mise sur le Marché) est à la France.

19. L'échinacée est une plante d'Amérique du Nord utilisée à la fois pour diminuer les symptômes et la durée du rhume ou de la grippe et pour les prévenir. Certains attribuent à la plante une action bénéfique sur le système immunitaire.

20. Une étude récente (Kronenberg et coll., 2006) a montré que ce sont les problèmes chroniques en particulier associés à la douleur (arthrite, maux de dos) qui entraînent le plus de recours aux MAC et aux PSN chez les Américaines d'origine chinoise.

21. Une étude menée au Viêt Nam a montré notamment que $37 \%$ des femmes qui utilisent un contraceptif n'ont pas choisi la pilule contraceptive à cause des effets secondaires (Nguyen \& Vu, 2001).

22. Au vu de ces résultats préliminaires, nous avons l'intention de centrer une prochaine analyse sur les femmes de notre échantillon, une analyse qui insisterait sur l'articulation entre leurs problèmes de santé (en particulier problèmes allergiques et respiratoires, ménopause), les modèles explicatifs qu'elles en proposent et les choix thérapeutiques qu'elles font pour les solutionner en pays d'accueil. Il s'agira alors de mieux saisir non seulement l'impact différencié de l'expérience de l'immigration sur l'évolution des pratiques de santé de ce sous-groupe d'immigrants mais également d'affiner notre compréhension du pluralisme thérapeutique comme manifestation non pas forcément d'une adaptation et d'une intégration difficile mais plutôt d'un mouvement d'acculturation complexe. 


\section{RÉSUMÉS}

Cet article propose une réflexion renouvelée sur la problématique "santé - immigration » à partir d'une recherche qualitative sur les pratiques de recours au médicament des Vietnamiens immigrés de Montréal (Canada) et leurs déterminants socioculturels. Notre étude confirme l'existence de pratiques de pluralisme médical au sein de cette communauté mais, surtout, met en lumière la complexité et la variabilité des formes que prend ce pluralisme. Au travers de la reconstruction d'un certain nombre d'itinéraires thérapeutiques individuels, nous avons également pu identifier l'importance des représentations (du médicament, du corps, de la maladie, de la santé) entrant en jeu dans ces pratiques.

Culture, Immigration and Health. The Consumption of Medicines among Vietnamese People living in Montreal. Our aim in this paper is to contribute to renew the debate surrounding the "health - immigration" issue through qualitative research dealing with the recourse to medicines among Vietnamese immigrants in Montreal (Canada). Our analysis confirms the existence of practices of medical pluralism within the community. It also reveals the complexity and the variability of forms this medical pluralism would take. Through the rebuilding of some specific therapeutic itineraries, we also raise the role some cultural factors play - especially the cultural beliefs about health, disease, medicines, or the body - in the population's therapeutic choices and health practices.

Cultura, inmigración y salud. El consumo de medicamientos en los Vietnamitas de Montreal. Este artículo propone una reflexión renovada sobre la problemática «salud inmigración» a partir de una investigación cualitativa sobre el recurso al medicamento de los Vietnamitas immigrados de Montreal (Canada). Nuestro estudio confirma la existencia de prácticas de pluralismo médico en el seno de esta comunidad pero también la complejidad y la variabilidad de las formas que toma este pluralismo. A través de la reconstrucción de algunos itinerarios terapeúticos individuales, pudimos también levantar algunos factores culturales representaciones de la salud, del cuerpo, del medicamento y de la enfermedad - deque entraban en las prácticas de salud de la población al estudio.

\section{AUTEURS}

\section{MARIE-EVE BLANC}

Ph. D., agente de recherche, GRASP (Groupe de Recherche sur les Aspects Sociaux de la Santé et de la Prévention), Université de Montréal, C.P. 6128 Succ. Centre-ville, Montréal, Québec, Canada H3C 3J7 marie-eve.blanc@umontreal.ca

\section{LAURENCE MONNAIS}

Ph. D., professeur agrégé, Département Histoire, Chercheur, équipe MEOS/GRASP, Université de Montréal laurence.monnais-rousselot@umontreal.ca 